\title{
Overexpression of long non-coding RNA differentiation antagonizing non-protein coding RNA inhibits the proliferation, migration and invasion and promotes apoptosis of renal cell carcinoma
}

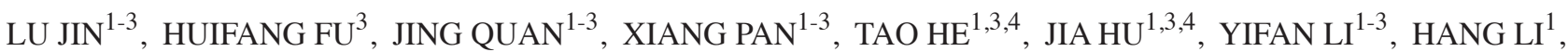 \\ YU YANG $^{1}$, JING YE ${ }^{3}$, FANGTING ZHANG ${ }^{3}$, LIANGCHAO NI ${ }^{1}$, SHANGQI YANG ${ }^{1,3}$ and YONGQING LAI ${ }^{1,3}$ \\ ${ }^{1}$ Department of Urology, Peking University Shenzhen Hospital, Shenzhen, Guangdong 518036; ${ }^{2}$ Department of Urology, \\ Anhui Medical University, Hefei, Anhui 230032; ${ }^{3}$ The Guangdong and Shenzhen Key Laboratory of \\ Male Reproductive Medicine and Genetics, Shenzhen, Guangdong 518036; ${ }^{4}$ Department of Urology, \\ Guangzhou Medical University, Guangzhou, Guangdong 511436, P.R. China
}

Received August 19, 2016; Accepted May 15, 2017

DOI: $10.3892 / \mathrm{mmr} .2017 .7135$

\begin{abstract}
Renal cell carcinoma (RCC) is the third most common cancer in the urological system; however, the pathogenesis remains unknown. Accumulating evidence has demonstrated that long non-coding RNAs are dysregulated in various tumors and serves an important role in tumorigenesis and development. In the present study, expression of IncRNA differentiation antagonizing non-protein coding RNA (DANCR) in 24 paired RCC and adjacent normal tissues was detected by reverse transcription-quantitative polymerase chain reaction. The results revealed that DANCR is downregulated in RCC tissues compared with adjacent normal tissues. Subsequent study revealed that overexpression of IncRNA DANCR by transfection of pcDNA3.1-DANCR could suppress 786-O and ACHN (RCC cell) proliferation, migration and invasion, and induce apoptosis compared with cells transfected with the pcDNA3.1 vector. The results revealed that DANCR functions as a tumor suppressor in RCC. In conclusion, the present study, to the best of our knowledge, was the first to reveal DANCR as a tumor suppressor. The results implicate DANCR as a potential biomarker of RCC, and further study will be focused on the clinical significance and signaling pathways of DANCR.
\end{abstract}

\section{Introduction}

Renal cell carcinoma (RCC) is a common malignant tumor occurring mainly in adults, which accounts for $3 \%$ of all malignant tumors in adults and $85 \%$ of all kidney tumors (1-3).

Correspondence to: Professor Yongqing Lai, Department of Urology, Peking University Shenzhen Hospital, 1120 Lianhua Road, Shenzhen, Guangdong 518036, P.R. China

E-mail: yqlord@163.com

Key words: long non-coding RNA, renal tumor, renal cell carcinoma, differentiation antagonizing non-protein coding RNA
RCC is the third most common cancer in urological system, after prostate and bladder cancer, and has the highest mortality rate $(4,5)$. Furthermore, RCC is resistant to both chemotherapy and radiotherapy, but not to surgery $(6,7)$. Patients are usually diagnosed for RCC when undergoing a medical examination or after having experienced some symptoms of RCC; currently, no biomarkers for the early diagnosis or targeted therapy of RCC exist. Therefore, it is necessary to improve the understanding of RCC and identify novel biomarkers for diagnosis, surveillance and targeted therapy for RCC.

Long non-coding RNA (lncRNA), at a length of $>200$ nucleotides, is identified as endogenous cellular RNAs lack of open reading frames of significant length $(<100$ amino acids) $(6,8)$. With the development of gene sequencing, more and more lncRNAs have been identified (9); however, the functions of most lncRNAs remain unclear. Previous studies have revealed that certain lncRNAs may regulate gene expression at various levels and serve important roles in tumorigenesis and development (10).

In the present study, the expression and function of lncRNA differentiation antagonizing non-protein coding RNA (DANCR) in RCC was investigated. A previous study revealed that IncRNA DANCR is upregulated in prostate cancer (11), hepatocellular carcinoma (HCC) (12) and colorectal cancer (CRC) (13). The present study detected the expression of DANCR in RCC tissues and adjacent paired normal tissues, and whether DANCR could affect the cellular function was also investigated.

\section{Materials and methods}

Sample collection. Paired RCC and adjacent normal tissues $(n=24)$ were used in the present study and were collected from Peking University Shenzhen Hospital (Shenzhen, China). Tissues were immersed in RNAlater (Qiagen $\mathrm{GmbH}$, Hilden, Germany) over $30 \mathrm{~min}$ while being dissected, then stored at $-80^{\circ} \mathrm{C}$ until further use. The adjacent normal tissues were $2 \mathrm{~cm}$ away from the RCC tissues and verified by pathological examination. All the tissues were reviewed and classified by 
hematoxylin and eosin (H\&E) staining. Collection and usage of the samples were approved by the Ethics Committees of Peking University Shenzhen Hospital. Written informed consent was obtained from all patients.

Cell culture and transfection. The 786-O and ACHN RCC cell lines (American Type Culture Collection, Manassas, VA, USA) and the HEK-293 cell line (Type Culture Collection of the Chinese Academy of Sciences, Shanghai, China) were used in this study. Cells were cultured at a temperature of $37^{\circ} \mathrm{C}$ in a humidified incubator containing $5 \% \mathrm{CO}_{2}$ in Dulbecco's modified Eagle's medium (DMEM; Gibco; Thermo Fisher Scientific, Inc., Waltham, MA, USA) supplemented with $10 \%$ fetal bovine serum, $1 \%$ antibiotics $(100 \mu \mathrm{l} / \mathrm{ml}$ penicillin and $100 \mathrm{mg} / \mathrm{ml} \mathrm{strep-}$ tomycin sulfates) and $1 \%$ glutamine (all from Gibco; Thermo Fisher Scientific, Inc.). Full-length lncRNA DANCR was subcloned into a pcDNA3.1 vector (GenePharma, Suzhou, China) and the vector (pcDNA3.1-DANCR) was verified by sequencing. For overexpression of lncRNA DANCR, synthesized pcDNA3.1-DANCR was transfected into cells using Lipofectamine 3000 (Invitrogen; Thermo Fisher Scientific, Inc.), which was mixed in the Opti-Minimum Essential Medium ${ }^{\circledR}$ I Reduced Serum Medium (Gibco; Thermo Fisher Scientific, Inc.) according to the manufacturer's protocol. Mock pcDNA3.1 served as the negative control. Reverse transcription-quantitative polymerase chain reaction (RT-qPCR) was performed to detect the expression of DANCR in cells after transfection.

RNA extraction, $c D N A$ synthesis and RT-qPCR. RNAiso Plus reagent (Takara Bio, Inc., Otsu, Japan) was used to extract RNA from tissues and cells. RT-qPCR was performed to obtain cDNA with $1 \mu \mathrm{g}$ RNA using a PrimeScript ${ }^{\mathrm{TM}}$ RT reagent kit (Takara Bio, Inc.). Expression of lncRNA was detected by performing qPCR with Premix Ex Taq ${ }^{\mathrm{TM}}$ II (Takara Bio, Inc.) on a Roche Light Cycler 480 Real-Time PCR system. Cycling conditions were as follows: An initial predenaturation step at $95^{\circ} \mathrm{C}$ for $1 \mathrm{~min}$, followed by 40 cycles of denaturation at $95^{\circ} \mathrm{C}$ for $10 \mathrm{sec}$, annealing at $60^{\circ} \mathrm{C}$ for $30 \mathrm{sec}$ and extension at $70^{\circ} \mathrm{C}$ for $30 \mathrm{sec}$. GAPDH was used as an internal control and sequences of primers are presented in Table I. The $\Delta \Delta \mathrm{Cq}$ method (14) was used to analyze the expression of DANCR in tissues and cells. Experiments were performed in triplicate.

Cell proliferation assay. Cell proliferation was assessed by performing MTT and Cell Counting kit-8 (CCK-8) assays. Approximately 3,000 cells/well were seeded into a 96-well plate, and $12 \mathrm{~h}$ later the cells were transfected with pcDNA-3.1-DANCR or mock. At 0, 24, 48 and $72 \mathrm{~h}$ after transfection, MTT or CCK-8 was added into the wells according to the manufacturer's protocol. The optical density (OD) value of each well was measured using an ELISA microplate reader (Bio-Rad Laboratories, Inc., Hercules, CA, USA) at a wave length of $490 \mathrm{~nm}$. The experiments were performed in six and repeated at least three times.

Cell mobility assay. Cell scratch and Transwell assays were performed to assess cell mobility ability of 786-O and ACHN cells. Approximately $3 \times 10^{5}$ cells/well were seeded into a 6-well plate and $24 \mathrm{~h}$ later were transfected with pcDNA3.1-DANCR
Table I. Primer sequences for real time-quantitative polymerase chain reaction.

Gene $\quad$ Sequence (5'-3')

\begin{tabular}{ll} 
DANCR & F: GCGCCACTATGTAGCGGGTT \\
& R: TCAATGGCTTGTGCCTGTAGTT \\
GAPDH & F: GGTCTCCTCTGACTTCAACA \\
& R: GTGAGGGTCTCTCTCTTCCT \\
\hline
\end{tabular}

F, forward; R, reverse; DANCR, long non-coding RNA differentiation antagonizing non-protein coding RNA.

or mock. At $6 \mathrm{~h}$ later, the cell monolayer was scratched with a sterile $200 \mu 1$ pipette tip to generate a line-shaped wound. Cells were then rinsed with phosphate-buffered saline to remove the floating cells, and then cells were cultured in DMEM supplemented with 5\% FBS. At 0 and $24 \mathrm{~h}$ later, scratches were imaged with a digital camera system. The experiments were performed in triplicate and repeated three times.

Transwell invasive and migratory assays were performed to assess the cell migratory and invasive ability. Transwell chamber inserts (BD Biosciences, Franklin Lakes, NJ, USA) with (for migration) or without (for invasion) Matrigel (BD Biosciences) were used according to the manufacturer's protocol. Transfected cells in $200 \mu \mathrm{l}$ DMEM were seeded at a density of $1 \times 10^{4}$ cells/well into the upper channel of the inserts. Cells were allowed to migrate for $36 \mathrm{~h}$ and invade for $48 \mathrm{~h}$. The cells that had migrated or invaded to the bottom of the inserts were stained with crystal violet and counted using a microscope. The experiments were performed in in triplicate and repeated at least three times.

Flow cytometric assay. Flow cytometry was performed to detect the apoptotic rate of cells after transfection, with Alexa Fluor $^{\circledR} 488$ Annexin V/Dead Cell Apoptosis kit (Invitrogen). After cells seeded for $24 \mathrm{~h}$, the cells would be transfected with pcDNA3.1-DANCR or mock. $48 \mathrm{~h}$ after transfection all the cells would be harvested and stained according to the manufacturer's advice. Then flow cytometry (Beckman, Brea, CA, USA) was used to analyze the apoptosis rate. The experiments were performed in in triplicate and repeated at least 3 times.

Bioinformatics. Prediction of miRNAs that may target lncRNA DANCR was determined using computational algorithms based on seed regions between the miRNAs and lncRNA DANCR. The miRDB database (http://mirdb. org/miRDB/index.html) was used in the present study to investigate the association between miRNAs and lncRNA DANCR.

Statistical analysis. Paired t-test was used to compare the expression levels of DANCR in matched tumor/normal tissues and cells. Student's t-test was used to analyze assays for characterizing phenotypes of cells. All the statistical analysis was carried out by SPSS 19.0 statistical software package (SPSS, Inc., Chicago, IL, USA). The results are expressed as the mean \pm standard deviation. $\mathrm{P}<0.05$ was considered to indicate a statistically significant difference. 


\section{Results}

LncRNA DANCR is downregulated in RCC tissues. qPCR was performed to detect the expression level of DANCR in tissues. The results $[\log 2$ ratio $(\mathrm{T} / \mathrm{N})]$ are presented in Fig. 1A, which indicated that DANCR is downregulated in 17 RCC tissues compared with adjacent normal tissues. The expression levels of DANCR in RCC and normal tissues are presented in Fig. 1B. The results demonstrated DANCR was significantly downregulated in RCC tissues compared with paired adjacent normal tissues $(\mathrm{P}<0.01)$.

Validation of the expression of IncRNA DANCR following transfection. Following transfection of pcDNA3.1-DANCR or mock (empty pcDNA3.1), qPCR was performed to detect the expression of DANCR. The results demonstrated that expression of lncRNA DANCR was 119.84 times (786-O cell, $\mathrm{P}<0.001$ ) and 175.73 times (ACHN cell, $\mathrm{P}<0.01$ ) higher in cells transfected with pcDNA3.1-DANCR than in cells transfected with the mock (Fig. 2).

Overexpression of DANCR suppresses cell proliferation. The results of the CCK- 8 and MTT assays are presented in Fig. 3. In 786-O cells, the proliferation ability was suppressed by 6.91\% (48 $\mathrm{h}, \mathrm{P}<0.01)$ and $8.56 \%(72 \mathrm{~h}, \mathrm{P}<0.01)$ for the CCK-8 assay (Fig. 3A), and by $7.30 \%$ (48 h, P<0.05) and $8.00 \%$ (72 h, $\mathrm{P}<0.001$ ) for the MTT assay, (Fig. 3B) in cells transfected with pcDNA3.1-DANCR compared with cells transfected with the mock. In ACHN cells, overexpression of DANCR suppressed the cell proliferative ability by $9.44 \%$ ( $24 \mathrm{~h}, \mathrm{P}<0.05), 10.24 \%$ (48 $\mathrm{h}, \mathrm{P}<0.01)$ and $12.04 \%(72 \mathrm{~h}, \mathrm{P}<0.001)$ for the CCK-8 assay (Fig. 3C) and by $7.74 \%$ (24 h, P<0.05), 9.86\% (48 h, P<0.01) and $9.03 \%$ (72 h, P<0.001) for the MTT assay (Fig. 3D). Therefore, the results revealed that 1 cRNA DANCR could inhibit RCC cell proliferation.

Overexpression of IncRNA DANCR inhibits RCC cell mobility. Cell scratch, Transwell migratory and Transwell invasive assays were performed to assess cell mobility ability. The results of the cell scratch assay revealed that overexpression of DANCR suppressed cell mobility ability by $25.38 \%$ for $786-\mathrm{O}$ cells $(\mathrm{P}<0.01$; Fig. 4A) and $60.47 \%$ for $\mathrm{ACHN}$ cells $(\mathrm{P}<0.01$; Fig. 4B).

The results of the Transwell assay demonstrated that in 786-O cells, overexpression of DANCR suppressed the cell invasive ability by $32.10 \%(\mathrm{P}<0.001)$ and the migratory ability by $34.13 \%$ ( $\mathrm{P}<0.001$; Fig. $5 \mathrm{~A})$. In ACHN cells invasive ability was reduced by $31.03 \%(\mathrm{P}<0.001)$ and migratory ability was reduced by $32.84 \%(\mathrm{P}<0.001$; Fig. 5B). The results indicated that IncRNA DANCR could suppress RCC cell mobility.

Overexpression of IncRNA DANCR induces cell apoptosis. The apoptotic rate was determined by flow cytometry. The results of flow cytometry indicated that apoptotic rate in 786-O cells transfected with pcDNA3.1-DANCR or mock was 41.03 vs. $11.58 \%$, respectively $(\mathrm{P}<0.01$, Fig. 6A). In ACHN cells, the apoptosis rate of cells transfected with pcDNA3.1-DANCR or mock was 32.48 vs. $14.76 \%$, respectively ( $\mathrm{P}<0.01$, Fig. $6 \mathrm{~B})$. The results revealed that DANCR could induce cell apoptosis in RCC.
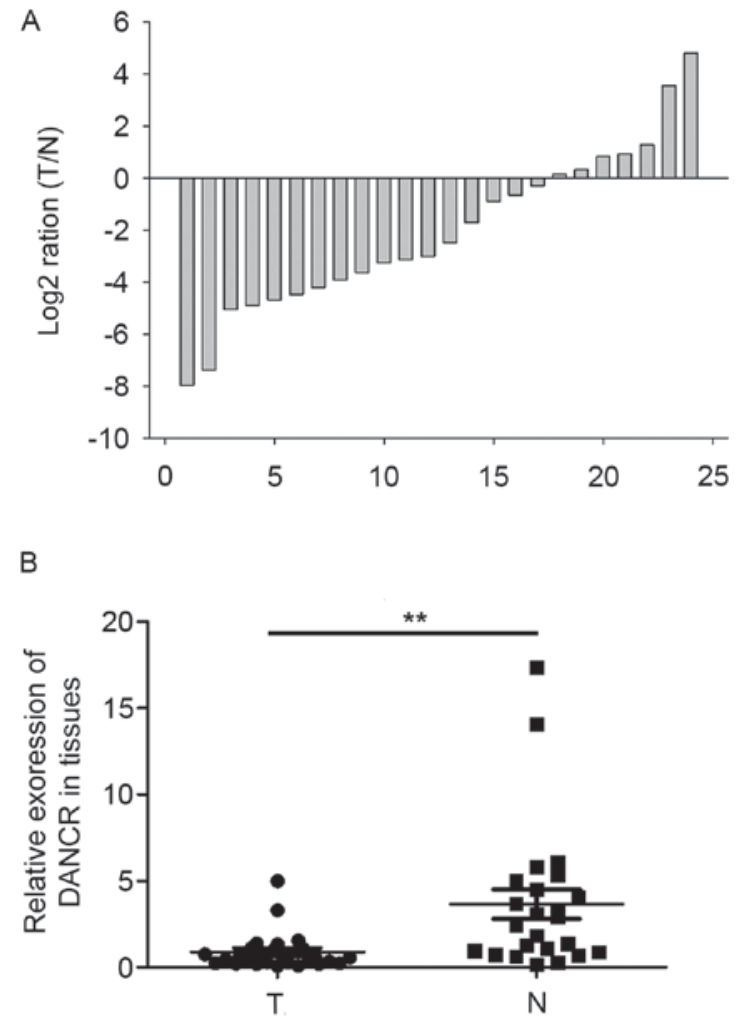

Figure 1. Expression of long non-coding RNA DANCR in tissues. (A) Log2 ratios $(\mathrm{T} / \mathrm{N})$ of DANCR in 24 paired tissues. DANCR was downregulated in 17 RCC tissues. (B) DANCR was downregulated in RCC tissues compared with normal tissues. Data are presented as the mean \pm standard deviation. ${ }^{* *} \mathrm{P}<0.01$. T, RCC tissues; N, normal tissues; DANCR, long non-coding RNA differentiation antagonizing non-protein coding RNA.

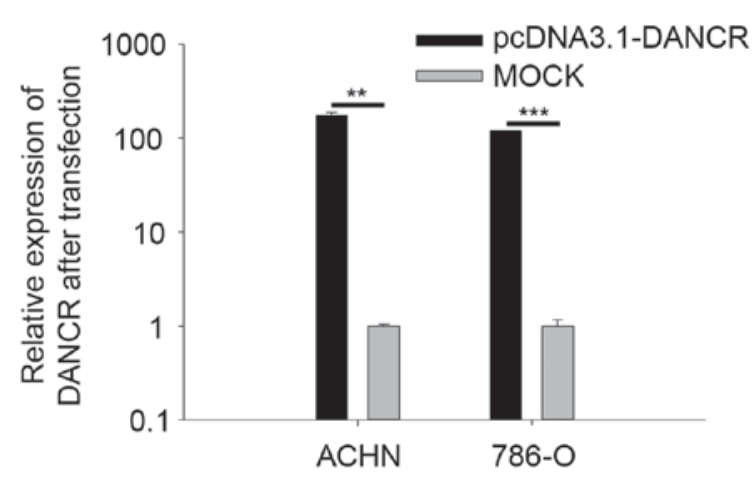

Figure 2. Relative expression of DANCR in ACHN and 786-O cells after transfection. Data are expressed as the mean \pm standard deviation. ${ }^{* *} \mathrm{P}<0.01$, ${ }^{* * *} \mathrm{P}<0.001$. DANCR, long non-coding RNA differentiation antagonizing non-protein coding RNA.

miRNA prediction. The results predicted by the miRDB database, 15 miRNAs were predicted to target lncRNA DANCR. The miRNAs with highest scores, miR-3646 and miR-634, have been demonstrated to be associated with tumorigenesis. Therefore, miR-3646 and miR-634 may be regulators of lncRNA DANCR.

\section{Discussion}

Accumulating evidence has indicated that lncRNAs are dysregulated in various tumors, are involved in most cellular 

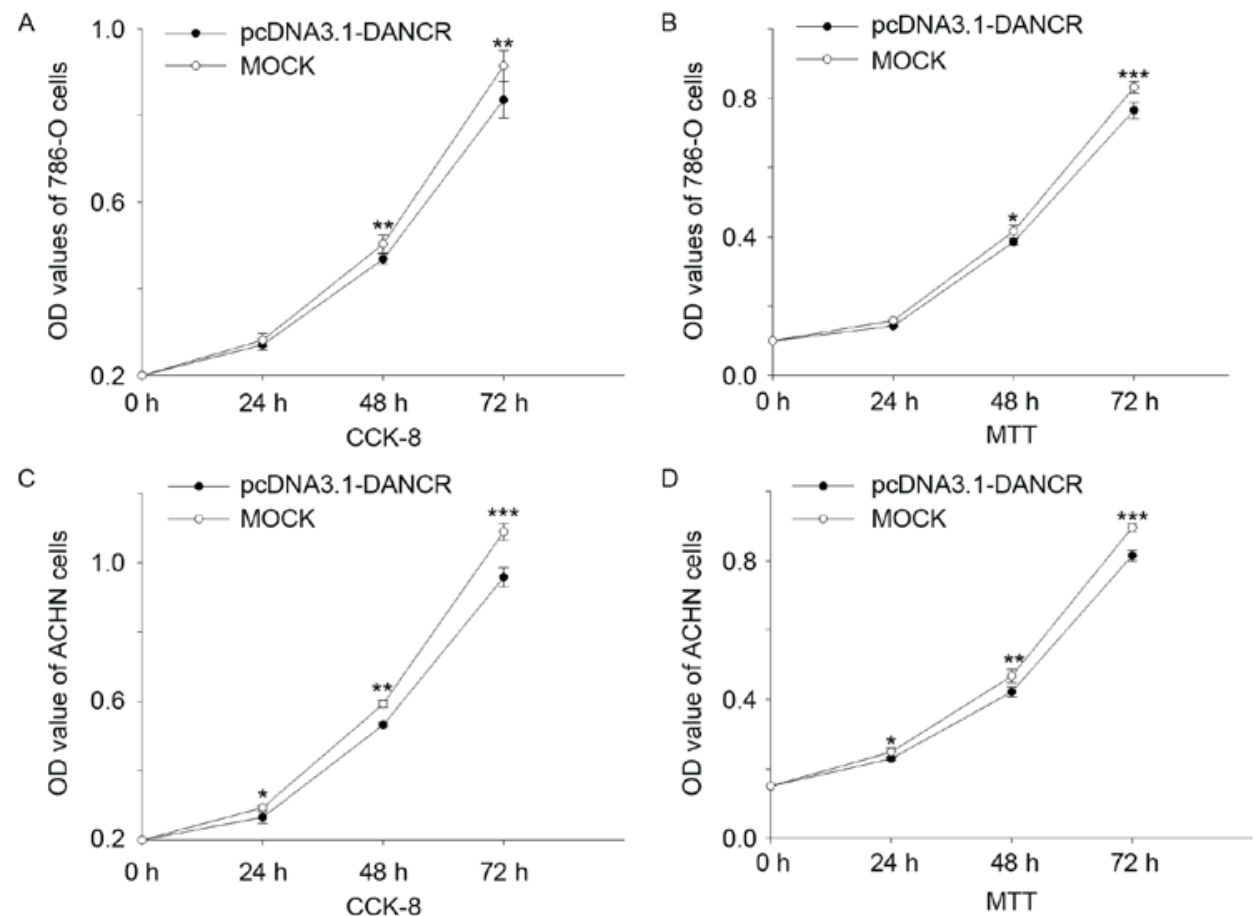

Figure 3. Cell proliferation assay of renal cell carcinoma cells transfected with pcDNA-3.1-DANCR or mock. (A) CCK-8 and (B) MTT assays of 786-O cells. (C) CCK-8 and (D) MTT assays of ACHN cells. Data are expressed as the mean \pm standard deviation. ${ }^{*} \mathrm{P}<0.05,{ }^{* *} \mathrm{P}<0.01,{ }^{* * *} \mathrm{P}<0.001$. DANCR, long non-coding RNA differentiation antagonizing non-protein coding RNA; CCK-8, Cell-Counting kit-8.
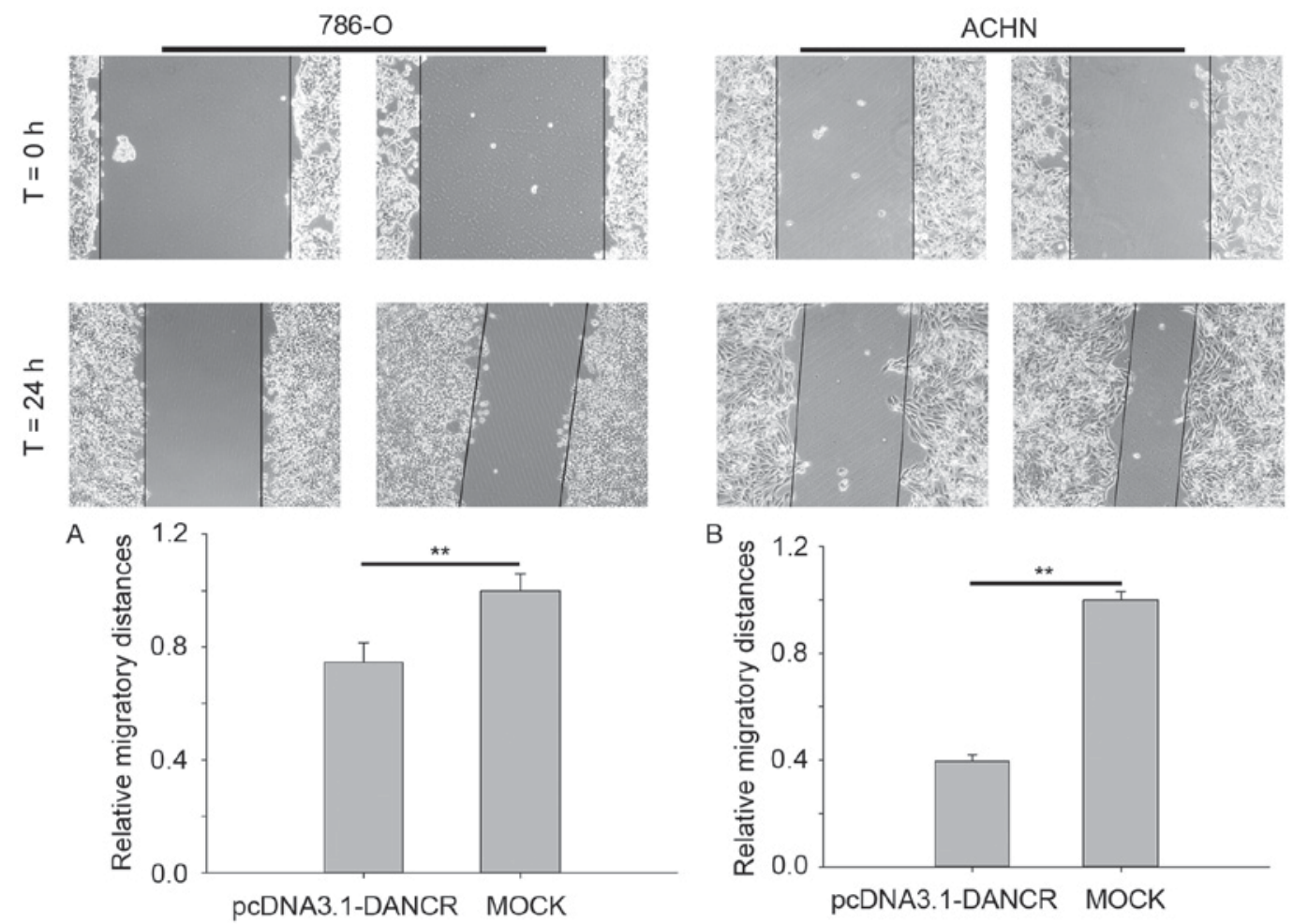

Figure 4. Cell scratch assay of 786-O and ACHN cells. Relative migratory distances of (A) 786-O and (B) ACHN cells following transfection of pcDNA-3.1-DANCR or mock. Data are expressed as the mean \pm standard deviation. ${ }^{* *} \mathrm{P}<0.01$. DANCR, long non-coding RNA differentiation antagonizing non-protein coding RNA; T, time.

processes $(15,16)$ and have marked tissue-specific expression features (17). A previous microarray study of RCC revealed 27,279 lncRNAs in RCC, of which 897 lncRNAs were dysregulated (18). In another study, Blondeau et al (19) identified
1,308 dysregulated lncRNAs in 15 clear cell RCCs by microarray. However, only a few IncRNAs have been studied and demonstrated to have some function in the tumorigenesis of RCC. 

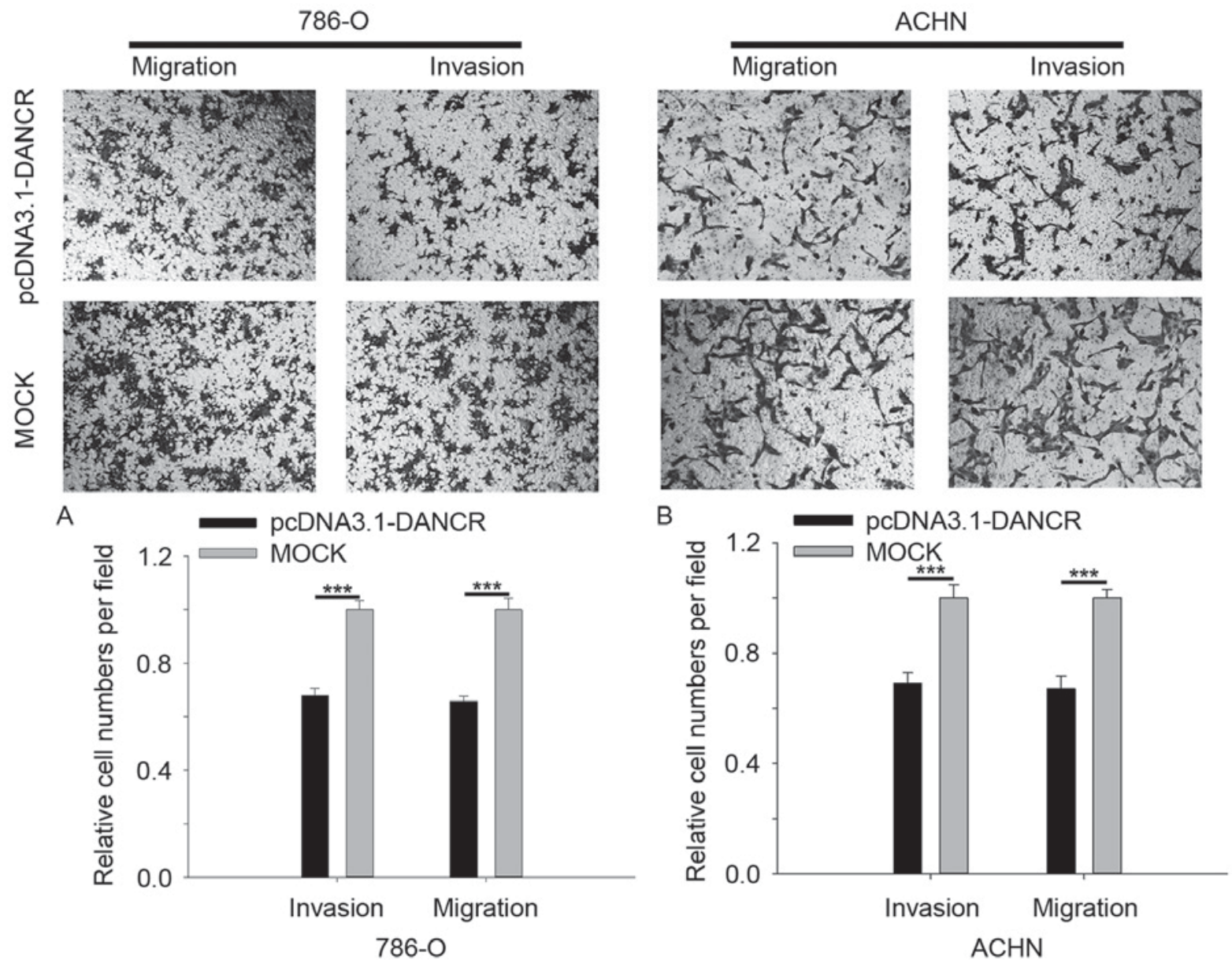

Figure 5. Over-expression of DANCR inhibits renal cell carcinoma cell migration and invasion. (A) 786-O and (B) ACHN cells. Data are expressed as the mean \pm standard deviation. ${ }^{* * * *} \mathrm{P}<0.001$. DANCR, long non-coding RNA differentiation antagonizing non-protein coding RNA.
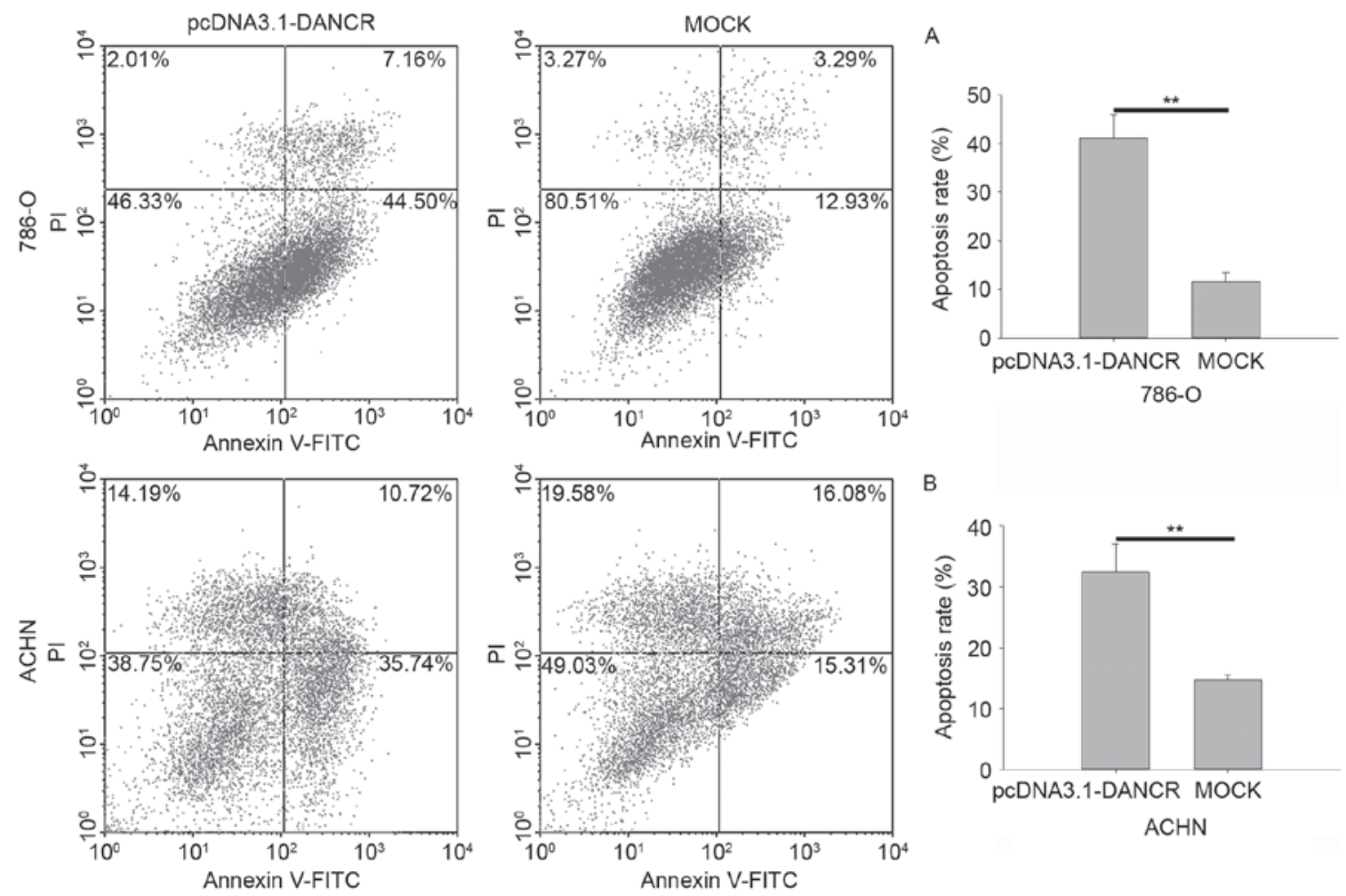

B

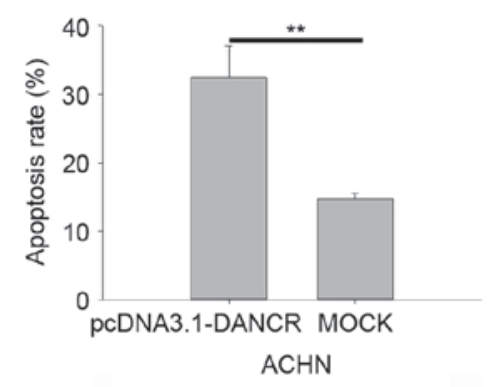

Figure 6. Over-expression of DANCR induces apoptosis of (A) 786-O and (B) ACHN cells. Data are expressed as the mean \pm standard deviation. ${ }^{* *} \mathrm{P}<0.01$. DANCR, long non-coding RNA differentiation antagonizing non-protein coding RNA; FITC, fluorescein isothiocyanate. 
lncRNA DANCR, 855 bp in length, was previously termed anti-differentiation non-coding RNA (ANCR) (20). A previous study revealed that IncRNA DANCR was upregulated in prostate cancer (11), hepatocellular carcinoma (12) and CRC (13). Jia et al (11) demonstrated that DANCR could promote the invasion of prostate cancer cells by silencing expression of tissue inhibitor of matrix metalloproteinase 2/3. In CRC, high expression of DANCR was reported to be associated with advanced tumor progression and poor prognosis (13), which indicated that DANCR may be a novel biomarker for CRC. Yuan et al (12) revealed that DANCR was upregulated in $\mathrm{HCC}$ and could increase the stemness features of HCC via upregulation of $\beta$-catenin. In the present study, DANCR was demonstrated to be downregulated in RCC tissues compared with adjacent normal tissues, which indicated that DANCR may have different functions in RCC. Subsequently experiments demonstrated that DANCR serves a role as a tumor suppressor in RCC by inhibiting RCC cell proliferation, migration and invasion, and inducing apoptosis. The database miRDB (http://mirdb.org/miRDB/index.html) predicted that microRNA (miR)-3646 and miR-634 might be regulators of DANCR. The associated miRNAs and downstream effectors require further investigation in future studies.

DANCR has also been demonstrated to serve an important role in non-cancer pathological processes. A study reported that DANCR could suppress odontoblast-like differentiation through inhibiting the Wnt/ $\beta$-catenin signaling pathway (21). Another study revealed that in patients with postmenopausal osteoporosis, DANCR was upregulated in blood monocytes and could negatively regulate interleukin- 6 and tumor necrosis factor- $\alpha$ expression (22). DANCR could also be an intermediary agent in some physiological processes. For example, SRY-box 4 could enhance chondrogenic differentiation and proliferation of human synovium-derived stem cell by activating DANCR (23).

In conclusion, the present study revealed that DANCR is downregulated in RCC tissues. From the in vitro experiments, overexpression of DANCR served an important role in inhibiting cellular proliferation, migration and invasion, and inducing apoptosis. To the best of our knowledge, DANCR was demonstrated as a tumor suppressor for the first time. Therefore, DANCR may represent a potential biomarker for RCC, and further studies should be focused on the clinical significance and the signaling pathway of DANCR.

\section{Acknowledgements}

The present study was supported by the National Natural Science Foundation of China (grant no. 81101922), the Science and Technology Development Fund Project of Shenzhen (grant nos. JCYJ20150403091443329 and JCYJ20170307111334308) and the fund of 'San-ming' Project of Medicine in Shenzhen and the fund of Guangdong Key Medical Subject (2015).

\section{References}

1. Slaby O, Jancovicova J, Lakomy R, Svoboda M, Poprach A, Fabian P, Kren L, Michalek J and Vyzula R: Expression of miRNA-106b in conventional renal cell carcinoma is a potential marker for prediction of early metastasis after nephrectomy. J Exp Clin Cancer Res 29: 90, 2010.
2. Tang $\mathrm{K}$ and $\mathrm{Xu} \mathrm{H}$ : Prognostic value of meta-signature miRNAs in renal cell carcinoma: An integrated miRNA expression profiling analysis. Sci Rep 5: 10272, 2015.

3. Zhai W, Sun Y, Jiang M, Wang M, Gasiewicz TA, Zheng J and Chang C: Differential regulation of LncRNA-SARCC suppresses VHL-mutant RCC cell proliferation yet promotes VHL-normal RCC cell proliferation via modulating androgen receptor/HIF-2 $\alpha /$ C-MYC axis under hypoxia. Oncogene 35: 4866-4880, 2016.

4. Bukowski RM: Prognostic factors for survival in metastatic renal cell carcinoma: Update 2008. Cancer 115 (10 Suppl): 2273-2281, 2009.

5. Cairns P: Renal cell carcinoma. Cancer Biomark 9: 461-473, 2010.

6. Qiao HP, Gao WS, Huo JX and Yang ZS: Long non-coding RNA GAS5 functions as a tumor suppressor in renal cell carcinoma. Asian Pac J Cancer Prev 14: 1077-1082, 2013.

7. Hirata H, Hinoda Y, Shahryari V, Deng G, Nakajima K, Tabatabai ZL, Ishii N and Dahiya R: Long noncoding RNA MALAT1 promotes aggressive renal cell carcinoma through Ezh2 and interacts with miR-205. Cancer Res 75: 1322-1331, 2015.

8. Gutschner T and Diederichs S: The hallmarks of cancer: A long non-coding RNA point of view. RNA Biol 9: 703-719, 2012.

9. Liu Z, Yan HY, Xia SY, Zhang C and Xiu YC: Downregulation of long non-coding RNA TRIM52-AS1 functions as a tumor suppressor in renal cell carcinoma. Mol Med Rep 13: 3206-3212, 2016.

10. Wang M, Huang T, Luo G, Huang C, Xiao XY, Wang L, Jiang GS and Zeng FQ: Long non-coding RNA MEG3 induces renal cell carcinoma cells apoptosis by activating the mitochondrial pathway. J Huazhong Univ Sci Technolog Med Sci 35: 541-545, 2015.

11. Jia J, Li F, Tang XS, Xu S, Gao Y, Shi Q, Guo W, Wang X, He D and Guo P: Long noncoding RNA DANCR promotes invasion of prostate cancer through epigenetically silencing expression of TIMP2/3. Oncotarget 7: 37868-37881, 2016.

12. Yuan SX, Wang J, Yang F, Tao QF, Zhang J, Wang LL, Yang Y, Liu H, Wang ZG, Xu QG, et al: Long noncoding RNA DANCR increases stemness features of hepatocellular carcinoma by derepression of CTNNB1. Hepatology 63: 499-511, 2016.

13. Liu Y, Zhang M, Liang L, Li J and Chen YX: Over-expression of lncRNA DANCR is associated with advanced tumor progression and poor prognosis in patients with colorectal cancer. Int J Clin Exp Pathol 8: 11480-11484, 2015.

14. Livak KJ and Schmittgen TD: Analysis of relative gene expression data using real-time quantitative PCR and the 2(-Delta Delta C(T)) Method. Methods 25: 402-408, 2001.

15. Wu Y, Wang YQ, Weng WW, Zhang QY, Yang XQ, Gan HL, Yang YS, Zhang PP, Sun MH, Xu MD and Wang CF: A serum-circulating long noncoding RNA signature can discriminate between patients with clear cell renal cell carcinoma and healthy controls. Oncogenesis 5: e192, 2016.

16. Li Y, Wang T, Li Y, Chen D, Yu Z, Jin L, Ni L, Yang S, Mao X, Gui Y and Lai Y: Identification of long-non coding RNA UCA1 as an oncogene in renal cell carcinoma. Mol Med Rep 13: 3326-3334, 2016.

17. Zhang M, Lu W, Huang Y, Shi J, Wu X, Zhang X, Jiang R, Cai Z and Wu S: Downregulation of the long noncoding RNA TUG1 inhibits the proliferation, migration, invasion and promotes apoptosis of renal cell carcinoma. J Mol Histol 47: 421-428, 2016.

18. Qin C, Han Z, Qian J, Bao M, Li P, Ju X, Zhang S, Zhang L, Li S, Cao Q, et al: Expression pattern of long non-coding RNAs in renal cell carcinoma revealed by microarray. PLoS One 9: e99372, 2014.

19. Blondeau JJ, Deng M, Syring I, Schrödter S, Schmidt D, Perner S, Müller SC and Ellinger J: Identification of novel long non-coding RNAs in clear cell renal cell carcinoma. Clin Epigenetics 7: 10, 2015.

20. Kretz M, Webster DE, Flockhart RJ, Lee CS, Zehnder A, LopezPajares V, Qu K, Zheng GX, Chow J, Kim GE, et al: Suppression of progenitor differentiation requires the long noncoding RNA ANCR. Genes Dev 26: 338-343, 2012.

21. Chen L, Song Z, Huang S, Wang R, Qin W, Guo J and Lin Z: lncRNA DANCR suppresses odontoblast-like differentiation of human dental pulp cells by inhibiting wnt/8.5-catenin pathway. Cell Tissue Res 364: 309-318, 2016.

22. Tong X, Gu PC, Xu SZ and Lin XJ: Long non-coding RNA-DANCR in human circulating monocytes: A potential biomarker associated with postmenopausal osteoporosis. Biosci Biotechnol Biochem 79: 732-737, 2015.

23. Zhang L, Chen S, Bao N, Yang C, Ti Y, Zhou L and Zhao J: Sox4 enhances chondrogenic differentiation and proliferation of human synovium-derived stem cell via activation of long noncoding RNA DANCR. J Mol Histol 46: 467-473, 2015. 